\title{
Vector-boson production at hadron colliders: hard-collinear coefficients at the NNLO
}

\author{
Stefano Catani ${ }^{1}$, Leandro Cieri $^{1}$, Daniel de Florian ${ }^{2}$, Giancarlo Ferrera $^{3}$, Massimiliano Grazzini ${ }^{4}$,a,b \\ ${ }^{1}$ INFN, Sezione di Firenze and Dipartimento di Fisica e Astronomia, Università di Firenze, 50019 Sesto Fiorentino, Florence, Italy \\ ${ }^{2}$ Departamento de Física, FCEYN, Universidad de Buenos Aires, (1428) Pabellón 1 Ciudad Universitaria, Capital Federal, Argentina \\ ${ }^{3}$ Dipartimento di Fisica, Università di Milano and INFN, Sezione di Milano, 20133 Milan, Italy \\ ${ }^{4}$ Institut für Theoretische Physik, Universität Zürich, 8057 Zürich, Switzerland
}

Received: 22 August 2012 / Published online: 3 November 2012

(C) Springer-Verlag Berlin Heidelberg and Società Italiana di Fisica 2012

\begin{abstract}
We consider QCD radiative corrections to vectorboson production in hadron collisions. We present the nextto-next-to-leading order (NNLO) result of the hard-collinear coefficient function for the all-order resummation of logarithmically enhanced contributions at small transverse momenta. The coefficient function controls NNLO contributions in resummed calculations at full next-to-next-toleading logarithmic accuracy. The same coefficient function is used in applications of the subtraction method to perform fully exclusive perturbative calculations up to NNLO.
\end{abstract}

The transverse-momentum $\left(q_{T}\right)$ distribution of systems with high invariant mass $M$ (Drell-Yan lepton pairs, vector boson(s), Higgs boson(s) and so forth) produced in hadronic collisions is important for physics studies within and beyond the Standard Model (SM).

The computation of these distributions in perturbative QCD is complicated by the presence of large logarithmic contributions of the form $\ln \left(M^{2} / q_{T}^{2}\right)$ that need to be resummed to all perturbative orders in the QCD coupling $\alpha_{S}$. The method to perform the resummation is known [1-8], including recent developments on the discovered and resummed effects $[9,10]$ due to helicity and azimuthal correlations in gluon fusion subprocesses. The structure of the resummed calculation is organized in a process-independent form that is controlled by a set of perturbative functions with computable 'resummation coefficients'. All the resummation coefficients that are process independent are known since some time [11-18] up to the second order in $\alpha_{\mathrm{S}}$, and the third-order coefficient $A^{(3)}$ has been obtained in

\footnotetext{
a e-mail: grazzini@physik.uzh.ch

${ }^{\mathrm{b}}$ On leave of absence from INFN, Sezione di Firenze, Sesto Fiorentino, Florence, Italy.
}

Ref. [19]. The complete computations of the second-order resummation coefficients have been carried out in Refs. [20] and [21] for two benchmark processes, namely, the production of the SM Higgs boson through gluon fusion and vector-boson production through the Drell-Yan (DY) mechanism of quark-antiquark annihilation. The explicit analytic expressions for the $\mathcal{O}\left(\alpha_{\mathrm{S}}^{2}\right)$ hard-collinear resummation coefficients in the case of SM Higgs boson production in the large- $m_{\text {top }}$ limit have been presented in Ref. [22]. This paper parallels Ref. [22]: we concentrate on single vectorboson production, and we present the corresponding analytic expressions of the second-order hard-collinear coefficient functions $\mathcal{H}^{(2)}$.

QCD predictions for vector-boson production at hadron colliders are important for present and forthcoming studies at the Tevatron and the LHC. Resummed calculations of the $q_{T}$ spectrum of vector bosons and of related observables are presented in Refs. [23-39]. Calculations for vectorboson production at the fully exclusive level with respect to the accompanying QCD radiation have been carried out in Refs. [21, 40-43] up to the next-to-next-to-leading order (NNLO) in perturbative QCD.

In this paper we compute the hard-collinear coefficient function $\mathcal{H}^{(2)}$ and, thus, the complete analytical expression of the NNLO cross section for vector-boson production in the small- $q_{T}$ region. These results have a twofold relevance, in the context of both resummed and fixed-order calculations.

The knowledge of $\mathcal{H}^{(2)}$ can be implemented in resummed calculations at full next-to-next-to-leading logarithmic (NNLL) order to achieve uniform NNLO accuracy in the small $-q_{T}$ region. In the case of vector-boson production, this implementation has been carried out in Ref. [33] by using the impact-parameter space resummation formalism developed in Refs. [44, 45]. This formalism enforces a unitarity constraint and thus it guarantees that (upon inclusion 
of $\mathcal{H}^{(2)}$ ) the resummed $q_{T}$ spectrum returns the complete NNLO total cross section after integration over $q_{T}$.

The subtraction method of Ref. [20] exploits the knowledge of transverse-momentum resummation coefficients at $\mathcal{O}\left(\alpha_{\mathrm{S}}^{2}\right)$ to perform NNLO calculations at the fully exclusive level. The Higgs boson coefficient functions presented in Ref. [22] were used in the numerical computations of Refs. [20, 46]. The coefficient functions presented in this paper are precisely those that are needed for the actual implementation of this subtraction method in DY-type processes: they are used in Refs. [21] and [47] for the NNLO numerical computations of vector-boson production and of associated production of a Higgs boson and a $W$ boson. The diphoton NNLO calculation of Ref. [48] also uses part of the results of the present paper to treat the quark-antiquark annihilation subprocess $q \bar{q} \rightarrow \gamma \gamma$.

This paper is organized as follows. We first introduce our notation and illustrate the NNLO calculation of the vectorboson cross section at small values of $q_{T}$. Then we recall the transverse-momentum resummation formalism. Finally, we present our NNLO results in analytic form and the relation with the $q_{T}$ resummation coefficients at $\mathcal{O}\left(\alpha_{\mathrm{S}}^{2}\right)$.

We briefly introduce the theoretical framework and our notation. We consider the production of a vector boson $V$ $\left(V=W^{ \pm}, Z\right.$ and/or $\left.\gamma^{*}\right)$ in hadron-hadron collisions. We use the narrow width approximation and we treat the vector boson as an on-shell particle with mass $M$. The QCD expression of the vector-boson transverse-momentum cross section ${ }^{1}$ is

$$
\begin{aligned}
& \frac{d \sigma}{d q_{T}^{2}}\left(q_{T}, M, s\right) \\
& =\sum_{a, b} \int_{0}^{1} d z_{1} \int_{0}^{1} d z_{2} f_{a / h_{1}}\left(z_{1}, M^{2}\right) f_{b / h_{2}}\left(z_{2}, M^{2}\right) \\
& \quad \times \frac{d \hat{\sigma}_{a b}}{d q_{T}^{2}}\left(q_{T}, M, \hat{s}=z_{1} z_{2} s ; \alpha_{\mathrm{S}}\left(M^{2}\right)\right)
\end{aligned}
$$

where $f_{a / h_{i}}\left(x, \mu_{F}^{2}\right)\left(a=q_{f}, \bar{q}_{f}, g\right)$ are the parton densities of the colliding hadrons $\left(h_{1}\right.$ and $\left.h_{2}\right)$ at the factorization scale $\mu_{F}$, and $d \hat{\sigma}_{a b} / d q_{T}^{2}$ are the partonic cross sections. The centre-of-mass energy of the two colliding hadrons is denoted by $s$, and $\hat{s}$ is the partonic centre-of-mass energy. We use parton densities as defined in the $\overline{\mathrm{MS}}$ factorization scheme, and $\alpha_{\mathrm{S}}\left(\mu_{R}^{2}\right)$ is the QCD running coupling at the renormalization scale $\mu_{R}$ in the $\overline{\mathrm{MS}}$ renormalization scheme. In Eq. (1) and throughout the paper, the arbitrary factorization and renormalization scales, $\mu_{F}$ and $\mu_{R}$, are set to be equal to the vector-boson mass $M$.

\footnotetext{
${ }^{1}$ If $V=\gamma^{*}$ or if the vector boson $V$ is not an on-shell particle, the transverse-momentum cross section $d \sigma / d q_{T}^{2}$ has to be replaced by the doubly differential distribution $M^{2} d \sigma / d M^{2} d q_{T}^{2}$, where $M$ is the invariant mass of $V$.
}

The partonic cross sections $d \hat{\sigma}_{a b} / d q_{T}^{2}$ are computable in QCD perturbation theory as power series expansions in $\alpha_{\mathrm{S}}\left(M^{2}\right)$. We are interested in the perturbative contributions that are large in the small- $q_{T}$ region $\left(q_{T} \ll M\right)$ and, eventually, singular in the limit $q_{T} \rightarrow 0$. To explicitly recall the perturbative structure of these enhanced terms at small $q_{T}$, we follow Ref. [22] and we introduce the cumulative partonic cross section: ${ }^{2}$

$$
\begin{aligned}
& \int_{0}^{Q_{0}^{2}} d q_{T}^{2} \frac{d \hat{\sigma}_{a b}}{d q_{T}^{2}}\left(q_{T}, M, \hat{s}=M^{2} / z ; \alpha_{\mathrm{S}}\left(M^{2}\right)\right) \\
& \quad \equiv \sum_{c=q_{f}, \bar{q}_{f^{\prime}}} z \sigma_{c \bar{c}, V}^{(0)} \hat{R}_{c \bar{c} \leftarrow a b}^{V}\left(z, M / Q_{0} ; \alpha_{\mathrm{S}}\left(M^{2}\right)\right),
\end{aligned}
$$

where the overall normalization of the function $\hat{R}^{V}$ is defined with respect to $\sigma_{q_{f} \bar{q}_{f^{\prime}}, V}^{(0)}$, which is the Born level cross section for the quark-antiquark annihilation subprocess $q_{f} \bar{q}_{f^{\prime}} \rightarrow V$ (the quark flavours $f$ and $f^{\prime}$ are equal if $\left.V=Z, \gamma^{*}\right)$. The partonic function $\hat{R}^{V}$ has the following perturbative expansion:

$$
\begin{aligned}
& \hat{R}_{c \bar{c} \leftarrow a b}^{V}\left(z, M / Q_{0} ; \alpha_{\mathrm{S}}\right) \\
& \quad=\delta_{c a} \delta_{\bar{c} b} \delta(1-z)+\sum_{n=1}^{\infty}\left(\frac{\alpha_{\mathrm{S}}}{\pi}\right)^{n} \hat{R}_{c \bar{c} \leftarrow a b}^{V(n)}\left(z, M / Q_{0}\right) .
\end{aligned}
$$

The next-to-leading order (NLO) and NNLO contributions to the cumulative cross section in Eq. (2) are determined by the functions $\hat{R}^{V(1)}$ and $\hat{R}^{V(2)}$, respectively. The small- $q_{T}$ region of the cross section $d \hat{\sigma}_{a b} / d q_{T}^{2}$ is probed by performing the limit $Q_{0} \ll M$ in Eq. (2). In this limit, the NLO and NNLO functions $\hat{R}^{V(1)}$ and $\hat{R}^{V(2)}$ have the following behaviour:

$$
\begin{aligned}
\hat{R}_{c \bar{c} \leftarrow a b}^{V(1)}\left(z, M / Q_{0}\right)= & l_{0}^{2} \hat{R}_{c \bar{c} \leftarrow a b}^{(1 ; 2)}(z)+l_{0} \hat{R}_{c \bar{c} \leftarrow a b}^{(1 ; 1)}(z) \\
& +\hat{R}_{c \bar{c} \leftarrow a b}^{(1 ; 0)}(z)+\mathcal{O}\left(Q_{0}^{2} / M^{2}\right),
\end{aligned}
$$

$$
\begin{aligned}
\hat{R}_{c \bar{c} \leftarrow a b}^{V(2)}\left(z, M / Q_{0}\right)= & l_{0}^{4} \hat{R}_{c \bar{c} \leftarrow a b}^{(2 ; 4)}(z)+l_{0}^{3} \hat{R}_{c \bar{c} \leftarrow a b}^{(2 ; 3)}(z) \\
& +l_{0}^{2} \hat{R}_{c \bar{c} \leftarrow a b}^{(2 ; 2)}(z)+l_{0} \hat{R}_{c \bar{c} \leftarrow a b}^{(2 ; 1)}(z) \\
& +\hat{R}_{c \bar{c} \leftarrow a b}^{(2 ; 0)}(z)+\mathcal{O}\left(Q_{0}^{2} / M^{2}\right),
\end{aligned}
$$

where $l_{0}=\ln \left(M^{2} / Q_{0}^{2}\right)$. In Eqs. (4) and (5), the powers of the large logarithm $l_{0}$ are produced by the singular (though integrable) behaviour of $d \hat{\sigma}_{a b} / d q_{T}^{2}$ at small values of $q_{T}$. The coefficients $\hat{R}^{(1 ; m)}$ (with $m \leq 2$ ) and $\hat{R}^{(2 ; m)}$ (with

\footnotetext{
${ }^{2}$ In our notation, the subscripts $c$ and $\bar{c}$ denote a quark and an antiquark (or vice versa) that do not necessarily have the same flavour. The flavour structure depends on the produced vector boson $V$ and it is (implicitly) specified by the specific form of the Born level cross section $\sigma_{c \bar{c}, V}^{(0)}$.
} 
$m \leq 4$ ) of the large logarithms are independent of $Q_{0}$; these coefficients depend on the partonic centre-of-mass energy $\hat{s}$ and, more precisely, they are functions of the energy fraction $z=M^{2} / \hat{s}$. As is well known (see also Eq. (7)), the logarithmic coefficients $\hat{R}^{(n ; m)}$ do not depend on the specific vector boson that is produced by $q \bar{q}$ annihilation and, therefore, we have removed the explicit superscript $V$ (i.e., $\left.\hat{R}^{V(n ; m)}=\hat{R}^{(n ; m)}\right)$.

In this paper we present the result of the computation of the cumulative cross section in Eq. (2) up to NNLO. The partonic calculation is performed in analytic form by neglecting terms of $\mathcal{O}\left(Q_{0}^{2} / M^{2}\right)$ in the limit $Q_{0} \ll M$. Therefore, we determine the coefficient functions $\hat{R}^{(n ; m)}(z)$ in Eqs. (4) and (5).

To perform our calculation, we follow the same method as used in Ref. [22] to evaluate the transverse-momentum cross section for Higgs boson production. The $q_{T}$ integration in Eq. (2) is thus rewritten in the following form:

$$
\begin{aligned}
& \int_{0}^{Q_{0}^{2}} d q_{T}^{2} \frac{d \hat{\sigma}_{a b}}{d q_{T}^{2}}\left(q_{T}, M, \hat{s} ; \alpha_{\mathrm{S}}\right) \\
& \equiv \int_{0}^{+\infty} d q_{T}^{2} \frac{d \hat{\sigma}_{a b}}{d q_{T}^{2}}\left(q_{T}, M, \hat{s} ; \alpha_{\mathrm{S}}\right) \\
& \quad-\int_{Q_{0}^{2}}^{+\infty} d q_{T}^{2} \frac{d \hat{\sigma}_{a b}}{d q_{T}^{2}}\left(q_{T}, M, \hat{s} ; \alpha_{\mathrm{S}}\right) \\
& =\hat{\sigma}_{a b}^{\text {(tot) }}\left(M, \hat{s} ; \alpha_{\mathrm{S}}\right) \\
& \quad-\int_{Q_{0}^{2}}^{\infty} d q_{T}^{2} \int_{-\infty}^{+\infty} d \hat{y} \frac{d \hat{\sigma}_{a b}}{d \hat{y} d q_{T}^{2}}\left(\hat{y}, q_{T}, M, \hat{s} ; \alpha_{\mathrm{S}}\right),
\end{aligned}
$$

where $\hat{\sigma}_{a b}^{(\text {tot })}$ is the vector-boson total (i.e. integrated over $q_{T}$ ) cross section and $d \hat{\sigma}_{a b} / d \hat{y} d q_{T}^{2}$ is the corresponding doubly differential cross section with respect to the transverse momentum and rapidity ( $\hat{y}$ is the rapidity of $V$ in the centre-of-mass frame of the two colliding partons $a$ and $b$ ) of the vector boson. The total cross section $\hat{\sigma}_{a b}^{\text {(tot) }}\left(M, \hat{s} ; \alpha_{\mathrm{S}}\right)$ is known $[49,50]$ in analytic form up to NNLO (i.e., up to $\mathcal{O}\left(\alpha_{S}^{2} \sigma_{V}^{(0)}\right)$ ). In the region of large or, more precisely, non-vanishing values of $q_{T}$, the differential distribution $d \hat{\sigma}_{a b} / d \hat{y} d q_{T}^{2}$ is also known [51-53] in analytic form up to $\mathcal{O}\left(\alpha_{S}^{2} \sigma_{V}^{(0)}\right)$. Using these known results and exploiting Eq. (6), we can compute the cumulative partonic cross section up to the NNLO. Note that $q_{T}>Q_{0}$ in the last term on the right-hand side of Eq. (6). Therefore the corresponding integration of the expression $d \hat{\sigma}_{a b} / d \hat{y} d q_{T}^{2}$ [51-53] over $\hat{y}$ and $q_{T}^{2}$ is finite as long as $Q_{0} \neq 0$ : using the explicit expression of $d \hat{\sigma}_{a b} / d \hat{y} d q_{T}^{2}$ from ${ }^{3}$ Ref. [52], we carry out the integration in analytic from in the limit $Q_{0} \ll M$ (i.e.,

\footnotetext{
${ }^{3}$ We list some typos that we have found and corrected in some formulae of Ref. [52]. In Eq. (2.12), $B_{2}^{q G}$ has to be replaced by $B_{2}^{q G}+C_{2}^{q G}$,
}

we neglect terms of $\mathcal{O}\left(Q_{0}^{2} / M^{2}\right)$ on the right-hand side of Eq. (6)). The result of our calculation ${ }^{4}$ confirms the logarithmic structure in Eqs. (4) and (5), and it allows us to determine the NLO and NNLO coefficients $\hat{R}^{(1 ; m)}$ (with $m \leq 2$ ) and $\hat{R}^{(2 ; m)}$ (with $m \leq 4$ ) of the cumulative cross section in Eq. (2).

The results of the coefficient functions $\hat{R}^{(n ; m)}(z)$ are conveniently expressed in terms of transverse-momentum resummation coefficients. Therefore, before presenting the results, we recall how these functions are related to the perturbative coefficients of the transverse-momentum resummation formula for vector-boson production [7]. This relation also shows that from the knowledge of Eq. (5) we can fully determine the NNLO rapidity distribution of the vector boson in the small- $q_{T}$ region.

To present the transverse-momentum resummation formula, we first decompose the partonic cross section $d \hat{\sigma}_{a b} /$ $d q_{T}^{2}$ in Eq. (1) in the form $d \hat{\sigma}_{a b}=d \hat{\sigma}_{a b}^{\text {(sing) }}+d \hat{\sigma}_{a b}^{(\mathrm{reg})}$. The singular component, $d \hat{\sigma}_{a b}^{\text {(sing) }}$, contains all the contributions that are enhanced at small $q_{T}$. These contributions are proportional to $\delta\left(q_{T}^{2}\right)$ or to large logarithms of the type $1 / q_{T}^{2} \ln ^{m}\left(M^{2} / q_{T}^{2}\right)$. The remaining component, $d \hat{\sigma}_{a b}^{\text {(reg) }}$, of the partonic cross section is regular order-by-order in $\alpha_{\mathrm{S}}$ as $q_{T} \rightarrow 0$ : the integration of $d \hat{\sigma}_{a b}^{(\mathrm{reg})} / d q_{T}^{2}$ over the range $0 \leq q_{T} \leq Q_{0}$ leads to a result that, at each fixed order in $\alpha_{\mathrm{S}}$, it vanishes in the limit $Q_{0} \rightarrow 0$. Therefore, $d \hat{\sigma}_{a b}^{(\mathrm{reg})}$ only contributes to the terms of $\mathcal{O}\left(Q_{0}^{2} / M^{2}\right)$ on the right-hand side of Eqs. (4) and (5).

Inserting the decomposition $d \hat{\sigma}_{a b}=d \hat{\sigma}_{a b}^{\text {(sing) }}+d \hat{\sigma}_{a b}^{\text {(reg) }}$ in Eq. (1), we obtain a corresponding decomposition, $d \sigma=d \sigma^{(\text {sing })}+d \sigma^{(\mathrm{reg})}$, of the hadronic cross section. The transverse-momentum resummation formula for the singular component of the $q_{T}$ cross section at fixed value of the rapidity $y$ (the rapidity is defined in the centre-of-mass frame of the two colliding hadrons) of the vector boson reads [7, 8]

$$
\begin{aligned}
& \frac{d \sigma^{\text {(sing) }}}{d y d q_{T}^{2}}\left(y, q_{T}, M, s\right) \\
& =\frac{M^{2}}{s} \sum_{c=q_{f}, \bar{q}_{f^{\prime}}} \sigma_{c \bar{c}, V}^{(0)} \int_{0}^{+\infty} d b \frac{b}{2} J_{0}\left(b q_{T}\right) S_{q}(M, b) \\
& \quad \times \sum_{a_{1}, a_{2}} \int_{x_{1}}^{1} \frac{d z_{1}}{z_{1}} \int_{x_{2}}^{1} \frac{d z_{2}}{z_{2}}\left[H^{F} C_{1} C_{2}\right]_{c \bar{c} ; a_{1} a_{2}} \\
& \quad \times f_{a_{1} / h_{1}}\left(x_{1} / z_{1}, b_{0}^{2} / b^{2}\right) f_{a_{2} / h_{2}}\left(x_{2} / z_{2}, b_{0}^{2} / b^{2}\right),
\end{aligned}
$$

and $C_{2}^{q G}$ has to be replaced by $C_{3}^{q G}$. In Eq. (A.4), two signs have to be changed: $B_{1}^{q G}$ has to be replaced by $-B_{1}^{q G}$, and $A^{q G}$ has to be replaced by $-A^{q G}$. In the first line of Eq. (A.10), the term $C_{F}\left(f_{u}-f_{s}-f_{t}\right)$ has to be replaced by $C_{A}\left(f_{u}-f_{s}-f_{t}\right)$.

${ }^{4}$ Some technical details related to the limit $Q_{0} \ll M$ are illustrated in Ref. [22]. 
where the kinematical variables $x_{i}(i=1,2)$ are $x_{1}=$ $e^{+y} M / \sqrt{s}$ and $x_{2}=e^{-y} M / \sqrt{s}$. The integration variable $b$ is the impact parameter, $J_{0}\left(b q_{T}\right)$ is the zeroth-order Bessel function, and $b_{0}=2 e^{-\gamma_{E}}\left(\gamma_{E}=0.5772 \ldots\right.$ is the Euler number) is a numerical coefficient. The symbol $\left[H^{F} C_{1} C_{2}\right]_{c \bar{c} ; a_{1} a_{2}}$ briefly denotes the following function of the longitudinal-momentum fractions $z_{1}$ and $z_{2}$ :

$$
\begin{aligned}
& {\left[H^{\mathrm{DY}} C_{1} C_{2}\right]_{c \bar{c} ; a_{1} a_{2}}} \\
& =H_{q}^{\mathrm{DY}}\left(\alpha_{\mathrm{S}}\left(M^{2}\right)\right) C_{c a_{1}}\left(z_{1} ; \alpha_{\mathrm{S}}\left(b_{0}^{2} / b^{2}\right)\right) \\
& \quad \times C_{\bar{c} a_{2}}\left(z_{2} ; \alpha_{\mathrm{S}}\left(b_{0}^{2} / b^{2}\right)\right),
\end{aligned}
$$

where $H_{q}^{\mathrm{DY}}\left(\alpha_{\mathrm{S}}\right)$ and $C_{c a}\left(z ; \alpha_{\mathrm{S}}\right)\left(c=q_{f}, \bar{q}_{f}\right)$ are perturbative functions of $\alpha_{\mathrm{S}}$ (see Eqs. (12)-(13)).

The quark form factor $S_{q}(M, b)$ in Eq. (7) is a processindependent quantity $[7,8,11-13]$. Its functional dependence on $M$ and $b$ is controlled by two perturbative functions, which are usually denoted as $A_{q}\left(\alpha_{\mathrm{S}}\right)$ and $B_{q}\left(\alpha_{\mathrm{S}}\right)$ (see, e.g., Ref. [10] that uses the same notation as in Eq. (7)). Their corresponding $n$th order perturbative coefficients are $A_{q}^{(n)}$ and $B_{q}^{(n)}$. The coefficients $A_{q}^{(1)}, B_{q}^{(1)}, A_{q}^{(2)}$ [11-13] and $B_{q}^{(2)}[14,15]$ are known: their knowledge fully determines the perturbative expression of $S_{q}(M, b)$ up to $\mathcal{O}\left(\alpha_{\mathrm{S}}^{2}\right)$.

The perturbative function $H_{q}^{\mathrm{DY}}\left(\alpha_{\mathrm{S}}\right)$ in Eq. (8) is process dependent, since it is directly related to the production mechanism of the vector boson through quark-antiquark annihilation. However, $H_{q}^{\mathrm{DY}}$ is independent of the specific type of vector boson $V\left(V=W^{ \pm}, Z, \gamma^{*}\right)$, and we have introduced the generic superscript DY.

The partonic functions $C_{q_{f} a}$ and $C_{\bar{q}_{f} a}$ in Eq. (8) are instead process independent, as a consequence of the universality features of QCD collinear radiation. Owing to their process independence, these partonic functions fulfil the following relations:

$$
\begin{aligned}
& C_{q_{f} q_{f^{\prime}}}\left(z ; \alpha_{\mathrm{S}}\right) \\
& \quad=C_{\bar{q}_{f} \bar{q}_{f^{\prime}}\left(z ; \alpha_{\mathrm{S}}\right)} \\
& \quad \equiv C_{q q}\left(z ; \alpha_{\mathrm{S}}\right) \delta_{f f^{\prime}}+C_{q q^{\prime}}\left(z ; \alpha_{\mathrm{S}}\right)\left(1-\delta_{f f^{\prime}}\right), \\
& \quad C_{q_{f} \bar{q}_{f^{\prime}}}\left(z ; \alpha_{\mathrm{S}}\right) \\
& \quad=C_{\bar{q}_{f} q_{f^{\prime}}}\left(z ; \alpha_{\mathrm{S}}\right) \\
& \quad \equiv C_{q \bar{q}}\left(z ; \alpha_{\mathrm{S}}\right) \delta_{f f^{\prime}}+C_{q \bar{q}^{\prime}}\left(z ; \alpha_{\mathrm{S}}\right)\left(1-\delta_{f f^{\prime}}\right), \\
& C_{q_{f} g}\left(z ; \alpha_{\mathrm{S}}\right)=C_{\bar{q}_{f} g}\left(z ; \alpha_{\mathrm{S}}\right) \equiv C_{q g}\left(z ; \alpha_{\mathrm{S}}\right),
\end{aligned}
$$

which are a consequence of charge conjugation invariance and flavour symmetry of QCD. The dependence of the matrix $C_{c a}$ on the parton labels is thus fully specified by the five independent quark functions $C_{q q}, C_{q q^{\prime}}, C_{q \bar{q}}, C_{q \bar{q}^{\prime}}$ and $C_{q g}$ on the right-hand side of Eqs. (9)-(11).

We recall that the function $H_{q}^{\mathrm{DY}}\left(\alpha_{\mathrm{S}}\right)$, the quark functions $C_{q a}\left(\alpha_{S}\right)$ and the perturbative function $B_{q}\left(\alpha_{S}\right)$ of the quark form factor are not separately computable in an unambiguous way. Indeed, these three functions are related by a renormalization-group symmetry [8] that follows from the $b$-space factorization structure of Eq. (7). The unambiguous definition of these three functions thus requires the specification $^{5}$ of a resummation scheme [8]. Note, however, that considering the perturbative expansion ${ }^{6}$ of Eq. (7) (i.e., the perturbative expansion of the singular component of the $q_{T}$ cross section), the resummation-scheme dependence exactly cancels order-by-order in $\alpha_{\mathrm{S}}$.

The perturbative expansion of the quark functions $C_{q a}\left(\alpha_{\mathrm{S}}\right)$ and of the vector-boson function $H_{q}^{\mathrm{DY}}\left(\alpha_{\mathrm{S}}\right)$ is defined as follows:

$$
\begin{aligned}
& C_{q a}\left(z ; \alpha_{\mathrm{S}}\right)=\delta_{q a} \delta(1-z)+\sum_{n=1}^{\infty}\left(\frac{\alpha_{\mathrm{S}}}{\pi}\right)^{n} C_{q a}^{(n)}(z), \\
& \left(a=g, q, \bar{q}, q^{\prime}, \bar{q}^{\prime}\right), \\
& H_{q}^{\mathrm{DY}}\left(\alpha_{\mathrm{S}}\right)=1+\sum_{n=1}^{\infty}\left(\frac{\alpha_{\mathrm{S}}}{\pi}\right)^{n} H_{q}^{\mathrm{DY}(n)} .
\end{aligned}
$$

The first-order coefficient function $C_{q g}^{(1)}(z)$ is independent of the resummation scheme; its expression is $[14,15]$

$C_{q g}^{(1)}(z)=\frac{1}{2} z(1-z)$.

The first-order coefficients $C_{q q^{\prime}}^{(1)}(z), C_{q \bar{q}}^{(1)}(z)$ and $C_{q \bar{q}^{\prime}}^{(1)}(z)$ vanish,

$C_{q q^{\prime}}^{(1)}(z)=C_{q \bar{q}}^{(1)}(z)=C_{q \bar{q}^{\prime}}^{(1)}(z)=0$,

while the coefficients $C_{q q}^{(1)}(z)$ and $H_{q}^{\mathrm{DY}(1)}$ fulfil the following relation $[14,15,17,18]$ :

$$
\begin{aligned}
& C_{q q}^{(1)}(z)+\frac{1}{2} H_{q}^{\mathrm{DY}(1)} \delta(1-z) \\
& \quad=\frac{C_{F}}{2}\left(\left(\frac{\pi^{2}}{2}-4\right) \delta(1-z)+1-z\right) .
\end{aligned}
$$

The separate determination of $C_{q q}^{(1)}(z)$ and $H_{q}^{\mathrm{DY}(1)}$ requires the specification of a resummation scheme. For instance, considering the resummation scheme in which the coefficient $H_{q}^{\mathrm{DY}(1)}$ vanishes, the right-hand side of Eq. (16) gives the value of $C_{q q}^{(1)}(z)$, and the corresponding value of the

\footnotetext{
${ }^{5}$ The reader who is not interested in issues related to the specification of a resummation scheme can simply assume that $H_{q}^{\mathrm{DY}}\left(\alpha_{\mathrm{S}}\right) \equiv 1$ throughout this paper. The choice $H_{q}^{\mathrm{DY}}\left(\alpha_{\mathrm{S}}\right)=1$ is customarily used in most of the literature on $q_{T}$ resummation for vector-boson production. ${ }^{6}$ The resummation-scheme dependence also cancels by consistently expanding Eq. (7) in terms of classes of resummed (leading, next-toleading and so forth) logarithmic contributions [44].
} 
quark form factor coefficient $B_{q}^{(2)}$ is explicitly computed in Refs. $[14,15]$. The computation of the second-order coefficients $C_{q q}^{(2)}, C_{q q^{\prime}}^{(2)}, C_{q \bar{q}}^{(2)}, C_{q \bar{q}^{\prime}}^{(2)}, C_{q g}^{(2)}$ and $H_{q}^{\mathrm{DY}(2)}$ is the aim of the calculation described in this paper.

To the purpose of presenting the NNLO results for the cumulative cross section in Eq. (2), we also define the following hard-collinear coefficient function:

$$
\begin{aligned}
\mathcal{H}_{q \bar{q} \leftarrow a b}^{\mathrm{DY}}\left(z ; \alpha_{\mathrm{S}}\right) \equiv & H_{q}^{\mathrm{DY}}\left(\alpha_{\mathrm{S}}\right) \int_{0}^{1} d z_{1} \int_{0}^{1} d z_{2} \delta\left(z-z_{1} z_{2}\right) \\
& \times C_{q a}\left(z_{1} ; \alpha_{\mathrm{S}}\right) C_{\bar{q} b}\left(z_{2} ; \alpha_{\mathrm{S}}\right),
\end{aligned}
$$

which is directly related to the coefficient function in Eq. (8). The function $\mathcal{H}^{\text {DY }}$ depends only on the energy fraction $z$, and it arises after integration of the resummation formula (7) over the rapidity of the vector boson. Note that $\mathcal{H}^{\mathrm{DY}}$ is independent of the resummation scheme [8]. The perturbative expansion of the function $\mathcal{H}^{\text {DY }}$ directly follows from Eqs. (12)-(13). We have

$$
\begin{aligned}
\mathcal{H}_{q \bar{q} \leftarrow a b}^{\mathrm{DY}}\left(z ; \alpha_{\mathrm{S}}\right)= & \delta_{q a} \delta_{\bar{q} b} \delta(1-z) \\
& +\sum_{n=1}^{\infty}\left(\frac{\alpha_{\mathrm{S}}}{\pi}\right)^{n} \mathcal{H}_{q \bar{q} \leftarrow a b}^{\mathrm{DY}(n)}(z),
\end{aligned}
$$

where the first-order and second-order contributions are

$$
\begin{aligned}
\mathcal{H}_{q \bar{q} \leftarrow a b}^{\mathrm{DY}(1)}(z)= & \delta_{q a} \delta_{\bar{q} b} \delta(1-z) H_{q}^{\mathrm{DY}(1)} \\
& +\delta_{q a} C_{\bar{q} b}^{(1)}(z)+\delta_{\bar{q} b} C_{q a}^{(1)}(z), \\
\mathcal{H}_{q \bar{q} \leftarrow a b}^{\mathrm{DY}(2)}(z)= & \delta_{q a} \delta_{\bar{q} b} \delta(1-z) H_{q}^{\mathrm{DY}(2)}+\delta_{q a} C_{\bar{q} b}^{(2)}(z) \\
& +\delta_{\bar{q} b} C_{q a}^{(2)}(z) \\
& +H_{q}^{\mathrm{DY}(1)}\left(\delta_{q a} C_{\bar{q} b}^{(1)}(z)+\delta_{\bar{q} b} C_{q a}^{(1)}(z)\right) \\
& +\left(C_{q a}^{(1)} \otimes C_{\bar{q} b}^{(1)}\right)(z) .
\end{aligned}
$$

In Eq. (20) and in the following, the symbol $\otimes$ denotes the convolution integral (i.e., we define $(g \otimes h)(z) \equiv$ $\left.\int_{0}^{1} d z_{1} \int_{0}^{1} d z_{2} \delta\left(z-z_{1} z_{2}\right) g\left(z_{1}\right) h\left(z_{2}\right)\right)$.

In the limit $Q_{0} \ll M$, the perturbative expansion of the cumulative partonic cross section in Eq. (2) can directly be related to the resummation coefficients of Eq. (7). We refer the reader to Ref. [22] for a concise illustration of this relation and to Ref. [44] for more technical details. The NLO and NNLO functions $\hat{R}^{V(1)}$ and $\hat{R}^{V(2)}$ in Eqs. (4) and (5) have the following expressions:

$$
\begin{aligned}
\hat{R}_{q \bar{q} \leftarrow a b}^{V(1)}\left(z, M / Q_{0}\right) & \\
= & l_{0}^{2} \Sigma_{q \bar{q} \leftarrow a b}^{\mathrm{DY}(1 ; 2)}(z)+l_{0} \Sigma_{q \bar{q} \leftarrow a b}^{\mathrm{DY}(1 ; 1)}(z) \\
& +\mathcal{H}_{q \bar{q} \leftarrow a b}^{\mathrm{DY}(1)}(z)+\mathcal{O}\left(Q_{0}^{2} / M^{2}\right),
\end{aligned}
$$

$$
\begin{aligned}
\hat{R}_{q \bar{q} \leftarrow a b}^{V(2)}\left(z, M / Q_{0}\right) & \\
= & l_{0}^{4} \Sigma_{q \bar{q} \leftarrow a b}^{\mathrm{DY}(2 ; 4)}(z)+l_{0}^{3} \Sigma_{q \bar{q} \leftarrow a b}^{\mathrm{DY}(2 ; 3)}(z)+l_{0}^{2} \Sigma_{q \bar{q} \leftarrow a b}^{\mathrm{DY}(2 ; 2)}(z) \\
& +l_{0}\left(\Sigma_{q \bar{q} \leftarrow a b}^{\mathrm{DY}(2 ; 1)}(z)-16 \zeta_{3} \Sigma_{q \bar{q} \leftarrow a b}^{\mathrm{DY}(2 ; 4)}(z)\right) \\
& +\left(\mathcal{H}_{q \bar{q} \leftarrow a b}^{\mathrm{DY}(2)}(z)-4 \zeta_{3} \Sigma_{q \bar{q} \leftarrow a b}^{\mathrm{DY}(2 ; 3)}(z)\right)+\mathcal{O}\left(Q_{0}^{2} / M^{2}\right),
\end{aligned}
$$

where we have used the same notation as in Ref. [44]. The explicit expressions of the coefficient functions $\Sigma_{q \bar{q} \leftarrow a b}^{\mathrm{DY}(n ; m)}(z)$ in terms of the resummation coefficients are given in Eqs. (63), (64), (66)-(69) of Ref. [44] (we have to set $\mu_{R}=\mu_{F}=Q=M$, where $\mu_{R}, \mu_{F}$ and $Q$ are the auxiliary scales of Ref. [44]) and are not reported here. The coefficients $\mathcal{H}_{q \bar{q} \leftarrow a b}^{\mathrm{DY}(1)}$ and $\mathcal{H}_{q \bar{q} \leftarrow a b}^{\mathrm{DY}(2)}$ are exactly those in Eqs. (19) and (20) (they are also given in Eqs. (65) and (70) of Ref. [44]) The first-order terms $\Sigma_{q \bar{q} \leftarrow a b}^{\mathrm{DY}(1 ; 2)}$ and $\Sigma_{q \bar{q} \leftarrow a b}^{\mathrm{DY}(1 ; 1)}$ depend on the quark form factor $S_{q}(M, b)$. The second-order terms $\Sigma_{q \bar{q} \leftarrow a b}^{\mathrm{DY}(2 ; m)}$ depend on $\mathcal{H}_{q \bar{q} \leftarrow a b}^{\mathrm{DY}(1)}$ and on the quark form factor $S_{q}(M, b)$ up to $\mathcal{O}\left(\alpha_{\mathrm{S}}^{2}\right)$. The numerical coefficient $\zeta_{3} \simeq 1.202 \ldots$ ( $\zeta_{k}$ is the Riemann $\zeta$-function) on the righthand side of Eq. (22) originates from the Bessel transformations (see, e.g., Eqs. (B.18) and (B.30) in Appendix B of Ref. [44]).

We now document our results of the NNLO computation of the cumulative partonic cross section. Using Eqs. (21) and (22), the results for $\hat{R}^{V(1)}$ and $\hat{R}^{V(2)}$ allow us to extract $\Sigma^{\mathrm{DY}(n ; m)}$ and $\mathcal{H}^{\mathrm{DY}(n)}$ up to $\mathcal{O}\left(\alpha_{\mathrm{S}}^{2}\right)$. The explicit result of the NLO function $\hat{R}^{V(1)}(z)$ confirms the expressions of $\Sigma_{q \bar{q} \leftarrow a b}^{\mathrm{DY}(1 ; 2)}(z), \Sigma_{q \bar{q} \leftarrow a b}^{\mathrm{DY}(1 ; 1)}(z)$ and $\mathcal{H}_{q \bar{q} \leftarrow a b}^{\mathrm{DY}(1)}(z)$, as predicted by the $q_{T}$ resummation coefficients at $\mathcal{O}\left(\alpha_{\mathrm{S}}\right)$. At NNLO, the present knowledge [11-15] of the $q_{T}$ resummation coefficients at $\mathcal{O}\left(\alpha_{S}^{2}\right)$ predicts the expressions of the terms $\Sigma_{q \bar{q} \leftarrow a b}^{\mathrm{DY}(2 ; m)}(z)$, with $m=1,2,3,4$. Our result for the NNLO function $\hat{R}^{V(2)}(z)$ confirms this prediction, and it allows us to extract the explicit expression of the second-order coefficient function $\mathcal{H}_{q \bar{q} \leftarrow a b}^{\mathrm{DY}(2)}(z)$.

We obtain

$$
\begin{aligned}
\mathcal{H}_{q \bar{q}(q)}^{\mathrm{DY}(2)}(z) & \\
= & C_{A} C_{F}\left\{\left(\frac{7 \zeta_{3}}{2}-\frac{101}{27}\right)\left(\frac{1}{1-z}\right)_{+}\right. \\
& +\left(\frac{59 \zeta_{3}}{18}-\frac{1535}{192}+\frac{215 \pi^{2}}{216}-\frac{\pi^{4}}{240}\right) \delta(1-z) \\
& +\frac{1+z^{2}}{1-z}\left(-\frac{\mathrm{Li}_{3}(1-z)}{2}+\mathrm{Li}_{3}(z)\right. \\
& -\frac{\operatorname{Li}_{2}(z) \log (z)}{2}-\frac{1}{2} \operatorname{Li}_{2}(z) \log (1-z)-\frac{1}{24} \log ^{3}(z) \\
& \left.-\frac{1}{2} \log ^{2}(1-z) \log (z)+\frac{1}{12} \pi^{2} \log (1-z)-\frac{\pi^{2}}{8}\right)
\end{aligned}
$$


$+\frac{1}{1-z}\left(-\frac{1}{4}\left(11-3 z^{2}\right) \zeta_{3}\right.$

$-\frac{1}{48}\left(-z^{2}+12 z+11\right) \log ^{2}(z)$

$\left.-\frac{1}{36}\left(83 z^{2}-36 z+29\right) \log (z)+\frac{\pi^{2} z}{4}\right)$

$+(1-z)\left(\frac{\operatorname{Li}_{2}(z)}{2}+\frac{1}{2} \log (1-z) \log (z)\right)$

$\left.+\frac{z+100}{27}+\frac{1}{4} z \log (1-z)\right\}$

$+C_{F} n_{F}\left\{\frac{14}{27}\left(\frac{1}{1-z}\right)_{+}\right.$

$+\frac{1}{864}\left(192 \zeta_{3}+1143-152 \pi^{2}\right) \delta(1-z)$

$+\frac{\left(1+z^{2}\right)}{72(1-z)} \log (z)(3 \log (z)+10)$

$\left.+\frac{1}{108}(-19 z-37)\right\}$

$+C_{F}^{2}\left\{\frac{1}{4}\left(-15 \zeta_{3}+\frac{511}{16}-\frac{67 \pi^{2}}{12}+\frac{17 \pi^{4}}{45}\right) \delta(1-z)\right.$

$+\frac{1+z^{2}}{1-z}\left(\frac{\mathrm{Li}_{3}(1-z)}{2}-\frac{5 \mathrm{Li}_{3}(z)}{2}\right.$

$+\frac{1}{2} \operatorname{Li}_{2}(z) \log (1-z)+\frac{3 \operatorname{Li}_{2}(z) \log (z)}{2}$

$+\frac{3}{4} \log (z) \log ^{2}(1-z)+\frac{1}{4} \log ^{2}(z) \log (1-z)$

$\left.-\frac{1}{12} \pi^{2} \log (1-z)+\frac{5 \zeta_{3}}{2}\right)$

$+(1-z)\left(-\mathrm{Li}_{2}(z)-\frac{3}{2} \log (1-z) \log (z)\right.$

$\left.+\frac{2 \pi^{2}}{3}-\frac{29}{4}\right)+\frac{1}{24}(1+z) \log ^{3}(z)$

$+\frac{1}{1-z}\left(\frac{1}{8}\left(-2 z^{2}+2 z+3\right) \log ^{2}(z)\right.$

$\left.+\frac{1}{4}\left(17 z^{2}-13 z+4\right) \log (z)\right)$

$\left.-\frac{z}{4} \log (1-z)\right\}$

$+C_{F}\left\{\frac{1}{z}(1-z)\left(2 z^{2}-z+2\right)\right.$

$\times\left(\frac{\mathrm{Li}_{2}(z)}{6}+\frac{1}{6} \log (1-z) \log (z)-\frac{\pi^{2}}{36}\right)$

$+\frac{1}{216 z}(1-z)\left(136 z^{2}-143 z+172\right)$ $-\frac{1}{48}\left(8 z^{2}+3 z+3\right) \log ^{2}(z)$

$+\frac{1}{36}\left(32 z^{2}-30 z+21\right) \log (z)$

$\left.+\frac{1}{24}(1+z) \log ^{3}(z)\right\}$,

$\mathcal{H}_{q \bar{q} \leftarrow q \bar{q}^{\prime}}^{\mathrm{DY}(2)}(z)$

$=C_{F}\left\{\frac{1}{12 z}(1-z)\left(2 z^{2}-z+2\right)\right.$

$\times\left(\operatorname{Li}_{2}(z)+\log (1-z) \log (z)-\frac{\pi^{2}}{6}\right)$

$+\frac{1}{432 z}(1-z)\left(136 z^{2}-143 z+172\right)$

$+\frac{1}{48}(1+z) \log ^{3}(z)-\frac{1}{96}\left(8 z^{2}+3 z+3\right) \log ^{2}(z)$

$\left.+\frac{1}{72}\left(32 z^{2}-30 z+21\right) \log (z)\right\}$

$\mathcal{H}_{q \bar{q} \leftarrow q q}^{\mathrm{DY}(2)}(z)=C_{F}\left(C_{F}-\frac{1}{2} C_{A}\right)\left\{\frac{1+z^{2}}{1+z}\left(\frac{3 \mathrm{Li}_{3}(-z)}{2}\right.\right.$

$+\mathrm{Li}_{3}(z)+\mathrm{Li}_{3}\left(\frac{1}{1+z}\right)-\frac{\mathrm{Li}_{2}(-z) \log (z)}{2}$

$-\frac{\mathrm{Li}_{2}(z) \log (z)}{2}-\frac{1}{24} \log ^{3}(z)-\frac{1}{6} \log ^{3}(1+z)$

$\left.+\frac{1}{4} \log (1+z) \log ^{2}(z)+\frac{\pi^{2}}{12} \log (1+z)-\frac{3 \zeta_{3}}{4}\right)$

$+(1-z)\left(\frac{\operatorname{Li}_{2}(z)}{2}+\frac{1}{2} \log (1-z) \log (z)+\frac{15}{8}\right)$

$-\frac{1}{2}(1+z)\left(\operatorname{Li}_{2}(-z)+\log (z) \log (1+z)\right)$

$\left.+\frac{\pi^{2}}{24}(z-3)+\frac{1}{8}(11 z+3) \log (z)\right\}$

$+C_{F}\left\{\frac{1}{12 z}(1-z)\left(2 z^{2}-z+2\right)\right.$

$\times\left(\operatorname{Li}_{2}(z)+\log (1-z) \log (z)-\frac{\pi^{2}}{6}\right)$

$+\frac{1}{432 z}(1-z)\left(136 z^{2}-143 z+172\right)$

$-\frac{1}{96}\left(8 z^{2}+3 z+3\right) \log ^{2}(z)$

$+\frac{1}{72}\left(32 z^{2}-30 z+21\right) \log (z)$

$\left.+\frac{1}{48}(1+z) \log ^{3}(z)\right\}$, 


$$
\begin{aligned}
& \mathcal{H}_{q \bar{q} \leftarrow q q^{\prime}}^{\mathrm{DY}(2)}(z)=\mathcal{H}_{q \bar{q} \leftarrow q \bar{q}^{\prime}}^{\mathrm{DY}(2)}(z), \\
& \mathcal{H}_{q \bar{q} \leftarrow q g}^{\mathrm{DY}(2)}(z) \\
& =C_{A}\left\{-\frac{1}{12 z}(1-z)\left(11 z^{2}-z+2\right) \operatorname{Li}_{2}(1-z)\right. \\
& +\left(2 z^{2}-2 z+1\right)\left(\frac{\operatorname{Li}_{3}(1-z)}{8}\right. \\
& \left.-\frac{1}{8} \operatorname{Li}_{2}(1-z) \log (1-z)+\frac{1}{48} \log ^{3}(1-z)\right) \\
& +\left(2 z^{2}+2 z+1\right)\left(\frac{3 \operatorname{Li}_{3}(-z)}{8}\right. \\
& +\frac{1}{32}\left(-8 z^{2}+23 z+8\right) \log (z) \\
& +\frac{5}{24} \pi^{2}(1-z) z+\frac{11 z}{32}+\frac{1}{8}(1-z) z \log ^{2}(1-z) \\
& -\frac{1}{4}(1-z) z \log (1-z) \log (z) \\
& \left.-\frac{1}{16}(3-4 z) z \log (1-z)-\frac{9}{32}\right\} \text {, } \\
& \mathcal{H}_{q \bar{q} \leftarrow g g}^{\mathrm{DY}(2)}(z)=-\frac{z}{2}\left(1-z+\frac{1}{2}(1+z) \log (z)\right),
\end{aligned}
$$$$
+\frac{\operatorname{Li}_{3}\left(\frac{1}{1+z}\right)}{4}-\frac{\operatorname{Li}_{2}(-z) \log (z)}{8}-\frac{1}{24} \log ^{3}(1+z)
$$$$
\left.+\frac{1}{16} \log ^{2}(z) \log (1+z)+\frac{1}{48} \pi^{2} \log (1+z)\right)
$$$$
+\frac{1}{4} z(1+z) \operatorname{Li}_{2}(-z)+z \operatorname{Li}_{3}(z)
$$$$
-\frac{1}{2} z \operatorname{Li}_{2}(1-z) \log (z)-z \operatorname{Li}_{2}(z) \log (z)
$$$$
-\frac{3}{8}\left(2 z^{2}+1\right) \zeta_{3}-\frac{149 z^{2}}{216}
$$$$
-\frac{1}{96}\left(44 z^{2}-12 z+3\right) \log ^{2}(z)
$$$$
+\frac{1}{72}\left(68 z^{2}+6 \pi^{2} z-30 z+21\right) \log (z)
$$$$
+\frac{\pi^{2} z}{24}+\frac{43 z}{48}+\frac{43}{108 z}+\frac{1}{48}(2 z+1) \log ^{3}(z)
$$$$
-\frac{1}{2} z \log (1-z) \log ^{2}(z)-\frac{1}{8}(1-z) z \log ^{2}(1-z)
$$$$
+\frac{1}{4} z(1+z) \log (1+z) \log (z)
$$$$
\left.+\frac{1}{16}(3-4 z) z \log (1-z)-\frac{35}{48}\right\}
$$$$
+C_{F}\left\{( 2 z ^ { 2 } - 2 z + 1 ) \left(\zeta_{3}-\frac{\mathrm{Li}_{3}(1-z)}{8}\right.\right.
$$$$
-\frac{\mathrm{Li}_{3}(z)}{8}+\frac{1}{8} \mathrm{Li}_{2}(1-z) \log (1-z)
$$$$
+\frac{\mathrm{Li}_{2}(z) \log (z)}{8}-\frac{1}{48} \log ^{3}(1-z)
$$$$
\left.+\frac{1}{16} \log (z) \log ^{2}(1-z)+\frac{1}{16} \log ^{2}(z) \log (1-z)\right)
$$$$
-\frac{3 z^{2}}{8}-\frac{1}{96}\left(4 z^{2}-2 z+1\right) \log ^{3}(z)
$$$$
+\frac{1}{64}\left(-8 z^{2}+12 z+1\right) \log ^{2}(z)
$$

where $C_{F}=\left(N_{c}^{2}-1\right) /\left(2 N_{c}\right), C_{A}=N_{c}\left(N_{c}\right.$ is the number of colours in $S U\left(N_{c}\right)$ QCD), $n_{F}$ is the number of quark flavours and $\mathrm{Li}_{k}(z)(k=2,3)$ are the usual polylogarithm functions,

$\operatorname{Li}_{2}(z)=-\int_{0}^{z} \frac{d t}{t} \ln (1-t)$,

$\mathrm{Li}_{3}(z)=\int_{0}^{1} \frac{d t}{t} \ln (t) \ln (1-z t)$.

We comment on the vector-boson results in Eqs. (23)(28) and on the ensuing determination of the second-order coefficients $C_{q q}^{(2)}, C_{q q^{\prime}}^{(2)}, C_{q \bar{q}}^{(2)}, C_{q \bar{q}^{\prime}}^{(2)}, C_{q g}^{(2)}$ and $H_{q}^{\mathrm{DY}(2)}$ in Eqs. (12) and (13).

The parton matrix $\mathcal{H}_{q \bar{q} \leftarrow a b}^{\mathrm{DY}(2)}$ is completely specified by the six entries ${ }^{7}$ in Eqs. (23)-(28): the quark-quark functions $\mathcal{H}_{q \bar{q} \leftarrow q \bar{q}}^{\mathrm{DY}(2)}, \mathcal{H}_{q \bar{q} \leftarrow q \bar{q}}^{\mathrm{DY}(2)}, \mathcal{H}_{q \bar{q} \leftarrow q q}^{\mathrm{DY}(2)}, \mathcal{H}_{q \bar{q} \leftarrow q q^{\prime}}^{\mathrm{DY}(2)}$, the quark-gluon function $\mathcal{H}_{q \bar{q} \leftarrow q g}^{\mathrm{DY}(2)}$ and the gluon-gluon function $\mathcal{H}_{q \bar{q} \leftarrow g g}^{\mathrm{DY}(2)}$.

Using Eq. (20), in the gluon-gluon channel we have

$\mathcal{H}_{q \bar{q} \leftarrow g g}^{\mathrm{DY}(2)}(z)=\left(C_{q g}^{(1)} \otimes C_{q g}^{(1)}\right)(z)$.

We see that the second-order coefficient function $\mathcal{H}_{q \bar{q} \leftarrow g g}^{\mathrm{DY}(2)}(z)$ is fully determined by the $q_{T}$ resummation coefficients at $\mathcal{O}\left(\alpha_{\mathrm{S}}\right)$. Using the value of $C_{q g}^{(1)}$ in Eq. (14), the expression on the right-hand side of Eq. (30) is in complete agreement with the result in Eq. (28). Therefore, our explicit computation of the NNLO partonic function $\hat{R}_{q \bar{q} \leftarrow g g}^{V(2)}$ represents a consistency check of the resummation formula (7).

Considering the quark-gluon channel, Eq. (20) can be recast in the following form:

$$
\begin{aligned}
& C_{q g}^{(2)}(z)+\frac{1}{2} H_{q}^{\mathrm{DY}(1)} C_{q g}^{(1)}(z) \\
& \quad=\mathcal{H}_{q \bar{q} \leftarrow q g}^{\mathrm{DY}(2)}(z)-\frac{1}{2}\left(\mathcal{H}_{q \bar{q} \leftarrow q \bar{q}}^{\mathrm{DY}(1)} \otimes C_{q g}^{(1)}\right)(z),
\end{aligned}
$$

\footnotetext{
${ }^{7}$ The other non-vanishing entries are obtained by the symmetry relation $\mathcal{H}_{q \bar{q} \leftarrow a b}^{\mathrm{DY}}=\mathcal{H}_{q \bar{q} \leftarrow \bar{b} \bar{a}}^{\mathrm{DY}}$. Several entries of the second-order matrix $\mathcal{H}_{q \bar{q} \leftarrow a b}^{\mathrm{DY}(2)}$ are vanishing because of Eq. (15).
} 
where we have used $\mathcal{H}_{q \bar{q} \leftarrow q \bar{q}}^{\mathrm{DY}(1)}(z)=H_{q}^{\mathrm{DY}(1)} \delta(1-z)+$ $2 C_{q q}^{(1)}(z)$ (see Eq. (19)). The relation (31) can be used to determine $C_{q g}^{(2)}(z)$ from the knowledge of $\mathcal{H}_{q \bar{q} \leftarrow q g}^{\mathrm{DY}(2)}$ and of the $q_{T}$ resummation coefficients at $\mathcal{O}\left(\alpha_{\mathrm{S}}\right)$. Inserting the firstorder results of Eqs. (14)-(16) in Eq. (31), we explicitly have

$$
\begin{aligned}
C_{q g}^{(2)}(z) & +\frac{1}{4} H_{q}^{\mathrm{DY}(1)} z(1-z) \\
= & \mathcal{H}_{q \bar{q} \leftarrow q g}^{\mathrm{DY}(2)}(z)-\frac{C_{F}}{4}\left[z \log (z)+\frac{1}{2}\left(1-z^{2}\right)\right. \\
& \left.+\left(\frac{\pi^{2}}{2}-4\right) z(1-z)\right],
\end{aligned}
$$

where $\mathcal{H}_{q \bar{q} \leftarrow q g}^{\mathrm{DY}(2)}$ is given in Eq. (27). Note that the right-hand side of Eq. (31) (or Eq. (32)) is resummation-scheme independent. Analogously to Eq. (16), the dependence of $C_{q g}^{(2)}$ on the resummation scheme is thus parametrized by the firstorder coefficient $H_{q}^{\mathrm{DY}(1)}$ on the left-hand side of Eq. (32).

The process-independent coefficient functions $C_{q q}^{(2)}(z)$, $C_{q q^{\prime}}^{(2)}(z) C_{q \bar{q}}^{(2)}(z)$ and $C_{q \bar{q}^{\prime}}^{(2)}(z)$ are obtained analogously to $C_{q g}^{(2)}(z)$. Considering the flavour diagonal quark-quark channel, Eq. (20) gives

$$
\begin{aligned}
2 C_{q q}^{(2)}(z) & +\delta(1-z)\left[H_{q}^{\mathrm{DY}(2)}-\frac{3}{4}\left(H_{q}^{\mathrm{DY}(1)}\right)^{2}\right] \\
& +\frac{1}{2} H_{q}^{\mathrm{DY}(1)} \mathcal{H}_{q \bar{q} \leftarrow q \bar{q}}^{\mathrm{DY}(1)}(z) \\
= & \mathcal{H}_{q \bar{q} \leftarrow q \bar{q}}^{\mathrm{DY}(2)}(z)-\frac{1}{4}\left(\mathcal{H}_{q \bar{q} \leftarrow q \bar{q}}^{\mathrm{DY}(1)} \otimes \mathcal{H}_{q \bar{q} \leftarrow q \bar{q}}^{\mathrm{DY}(1)}\right)(z),
\end{aligned}
$$

where the right-hand side of Eq. (33) is expressed in terms of resummation-scheme independent functions. Inserting Eqs. (14)-(16) in Eq. (33), we explicitly obtain

$$
\begin{aligned}
2 C_{q q}^{(2)}(z)+\delta(1-z)\left[H_{q}^{\mathrm{DY}(2)}-\frac{3}{4}\left(H_{q}^{\mathrm{DY}(1)}\right)^{2}\right. \\
\left.\quad+\frac{C_{F}}{4}\left(\pi^{2}-8\right) H_{q}^{\mathrm{DY}(1)}\right]+\frac{1}{2} C_{F} H_{q}^{\mathrm{DY}(1)}(1-z) \\
=\mathcal{H}_{q \bar{q} \leftarrow q \bar{q}}^{\mathrm{DY}(2)}(z)-\frac{C_{F}^{2}}{4}\left[\delta(1-z) \frac{\left(\pi^{2}-8\right)^{2}}{4}\right. \\
\left.\quad+\left(\pi^{2}-10\right)(1-z)-(1+z) \ln z\right],
\end{aligned}
$$

where $\mathcal{H}_{q \bar{q} \leftarrow q \bar{q}}^{\mathrm{DY}(2)}$ is given in Eq. (23). We observe that $C_{q q}^{(2)}(z)$ includes a resummation-scheme dependent part that depends on $H_{q}^{\mathrm{DY}(1)}$ and $H_{q}^{\mathrm{DY}(2)}$. We also recall [8] that the resummation-scheme invariance relates $C_{q q}^{(2)}, H_{q}^{\mathrm{DY}(2)}$ and the third-order coefficient $B_{q}^{(3)}$ of the quark form factor.
Considering the flavour off-diagonal quark-quark channel in Eq. (20), we obtain

$C_{q \bar{q}}^{(2)}(z)=\mathcal{H}_{q \bar{q} \leftarrow q q}^{\mathrm{DY}(2)}(z)$,

$C_{q q^{\prime}}^{(2)}(z)=\mathcal{H}_{q \bar{q} \leftarrow q \bar{q}^{\prime}}^{\mathrm{DY}(2)}(z)$,

$C_{q \bar{q}^{\prime}}^{(2)}(z)=\mathcal{H}_{q \bar{q} \leftarrow q q^{\prime}}^{\mathrm{DY}(2)}(z)$,

where $\mathcal{H}_{q \bar{q} \leftarrow q \bar{q}^{\prime}}^{\mathrm{DY}(2)}, \mathcal{H}_{q \bar{q} \leftarrow q q}^{\mathrm{DY}(2)}$, and $\mathcal{H}_{q \bar{q} \leftarrow q q^{\prime}}^{\mathrm{DY}(2)}$ are given in Eqs. (24)-(26). The off-diagonal second-order coefficients $C_{q \bar{q}}^{(2)}(z), C_{q q^{\prime}}^{(2)}(z)$ and $C_{q \bar{q}^{\prime}}^{(2)}(z)$ are resummation-scheme independent. From Eq. (26) we observe that we have $C_{q q^{\prime}}^{(2)}(z)=$ $C_{q \bar{q}^{\prime}}^{(2)}(z)$. The equality between $C_{q q^{\prime}}(z)$ and $C_{q \bar{q}^{\prime}}(z)$ is expected to be violated at higher perturbative orders (i.e., we expect $\left.C_{q q^{\prime}}^{(3)}(z) \neq C_{q \bar{q}^{\prime}}^{(3)}(z)\right)$.

In this paper we have considered QCD radiative corrections to vector-boson production in hadron-hadron collisions. We have presented the analytic result of the NNLO calculation of the vector-boson cross section at small values of $q_{T}$ (see Eqs. (2) and (5)). The NNLO result is compared (see Eq. (22)) with the predictions of transverse-momentum resummation. The comparison gives a second-order crosscheck of the all-order resummation formula (7), and it allows us to determine the previously unknown resummation coefficients at $\mathcal{O}\left(\alpha_{\mathrm{S}}^{2}\right)$. These are the coefficient functions $\mathcal{H}_{q \bar{q} \leftarrow a b}^{\mathrm{DY}(2)}(z)$ (see Eqs. (23)-(28)) and the related coefficients $C_{q g}^{(2)}, C_{q q}^{(2)}, C_{q q^{\prime}}^{(2)}(z), C_{q \bar{q}}^{(2)}(z)$ and $C_{q \bar{q}^{\prime}}^{(2)}(z)$ (see Eqs. (32), (34) and (35)), which control the dependence on the rapidity of the vector boson. The knowledge of these second-order coefficients is relevant for phenomenological applications of both resummed and fixed-order QCD computations. These coefficients have been already implemented in resummed calculations of the inclusive $q_{T}$ distribution at full NNLL accuracy [33]. Using the method of Ref. [20], the same coefficients have been used to perform the fully exclusive NNLO perturbative calculations of Refs. [21] and [47].

Acknowledgements This work was supported in part by UBACYT, CONICET, ANPCyT, INFN and the Research Executive Agency (REA) of the European Union under the Grant Agreement number PITN-GA-2010-264564 (LHCPhenoNet, Initial Training Network).

Note added Shortly after the Journal submission of this paper, Ref. [54] presented an NNLO calculation of the vector-boson transverse-momentum distribution at small $q_{T}$. In particular, the second-order quark-quark coefficient function $\mathcal{H}_{q \bar{q} \leftarrow q \bar{q}}^{D Y(2)}(z)$ or, equivalently, the quark-to-quark coefficient function $C_{q q}^{(2)}(z)$ (after having set $H_{q}^{D Y(1)}=H_{q}^{D Y(2)}=0$ ) is computed by using a method (based on transverse-momentum-dependent parton distributions) that is completely independent of the method used in our calculation. The result of $C_{q q}^{(2)}(z)$ obtained in Ref. [54] fully agrees with that in our Eq. (34). This agreement is a relevant crosscheck of the result of the two independent calculations. 


\section{References}

1. Y.L. Dokshitzer, D. Diakonov, S.I. Troian, Phys. Lett. B 79, 269 (1978)

2. Y.L. Dokshitzer, D. Diakonov, S.I. Troian, Phys. Rep. 58, 269 (1980)

3. G. Parisi, R. Petronzio, Nucl. Phys. B 154, 427 (1979)

4. G. Curci, M. Greco, Y. Srivastava, Nucl. Phys. B 159, 451 (1979)

5. J.C. Collins, D.E. Soper, Nucl. Phys. B 193, 381 (1981). [Erratumibid. B 213, 545 (1983)]

6. J.C. Collins, D.E. Soper, Nucl. Phys. B 197, 446 (1982)

7. J.C. Collins, D.E. Soper, G. Sterman, Nucl. Phys. B 250, 199 (1985)

8. S. Catani, D. de Florian, M. Grazzini, Nucl. Phys. B 596, 299 (2001)

9. P.M. Nadolsky, C. Balazs, E.L. Berger, C.-P. Yuan, Phys. Rev. D 76, 013008 (2007)

10. S. Catani, M. Grazzini, Nucl. Phys. B 845, 297 (2011)

11. J. Kodaira, L. Trentadue, Phys. Lett. B 112, 66 (1982)

12. J. Kodaira, L. Trentadue, Report SLAC-PUB-2934 (1982)

13. J. Kodaira, L. Trentadue, Phys. Lett. B 123, 335 (1983)

14. C.T.H. Davies, W.J. Stirling, Nucl. Phys. B 244, 337 (1984)

15. C.T.H. Davies, B.R. Webber, W.J. Stirling, Nucl. Phys. B 256, 413 (1985)

16. S. Catani, E. D'Emilio, L. Trentadue, Phys. Lett. B 211, 335 (1988)

17. D. de Florian, M. Grazzini, Phys. Rev. Lett. 85, 4678 (2000)

18. D. de Florian, M. Grazzini, Nucl. Phys. B 616, 247 (2001)

19. T. Becher, M. Neubert, Eur. Phys. J. C 71, 1665 (2011)

20. S. Catani, M. Grazzini, Phys. Rev. Lett. 98, 222002 (2007)

21. S. Catani, L. Cieri, G. Ferrera, D. de Florian, M. Grazzini, Phys. Rev. Lett. 103, 082001 (2009)

22. S. Catani, M. Grazzini, Eur. Phys. J. C 72, 2013 (2012)

23. C. Balazs, C.P. Yuan, Phys. Rev. D 56, 5558 (1997)

24. R.K. Ellis, D.A. Ross, S. Veseli, Nucl. Phys. B 503, 309 (1997)

25. J.-w. Qiu, X.-f. Zhang, Phys. Rev. D 63, 114011 (2001)

26. E.L. Berger, J.-w. Qiu, Phys. Rev. D 67, 034026 (2003)

27. E.L. Berger, J.-w. Qiu, Phys. Rev. Lett. 91, 222003 (2003)

28. A. Kulesza, W.J. Stirling, Eur. Phys. J. C 20, 349 (2001)

29. A. Kulesza, G.F. Sterman, W. Vogelsang, Phys. Rev. D 66, 014011 (2002)

30. F. Landry, R. Brock, P.M. Nadolsky, C.P. Yuan, Phys. Rev. D 67, 073016 (2003)
31. S. Berge, P.M. Nadolsky, F.I. Olness, Phys. Rev. D 73, 013002 (2006)

32. G. Bozzi, S. Catani, G. Ferrera, D. de Florian, M. Grazzini, Nucl. Phys. B 815, 174 (2009)

33. G. Bozzi, S. Catani, G. Ferrera, D. de Florian, M. Grazzini, Phys. Lett. B 696, 207 (2011)

34. S. Mantry, F. Petriello, Phys. Rev. D 84, 014030 (2011)

35. T. Becher, M. Neubert, D. Wilhelm, J. High Energy Phys. 1202, $124(2012)$

36. A. Banfi, M. Dasgupta, S. Marzani, L. Tomlinson, J. High Energy Phys. 1201, 044 (2012)

37. A. Banfi, M. Dasgupta, S. Marzani, L. Tomlinson, Report DCPT12-66. arXiv:1205.4760 [hep-ph]

38. A. Banfi, G.P. Salam, G. Zanderighi, J. High Energy Phys. 1206, $159(2012)$

39. A. Banfi, P.F. Monni, G.P. Salam, G. Zanderighi. Report CERNPH-TH-2012-173. arXiv:1206.4998 [hep-ph]

40. K. Melnikov, F. Petriello, Phys. Rev. Lett. 96, 231803 (2006)

41. K. Melnikov, F. Petriello, Phys. Rev. D 74, 114017 (2006)

42. R. Gavin, Y. Li, F. Petriello, S. Quackenbush, Comput. Phys. Commun. 182, 2388 (2011)

43. R. Gavin, Y. Li, F. Petriello, S. Quackenbush. Report ANL-HEPPR-11-83. arXiv:1201.5896 [hep-ph]

44. G. Bozzi, S. Catani, D. de Florian, M. Grazzini, Nucl. Phys. B 737, 73 (2006)

45. G. Bozzi, S. Catani, D. de Florian, M. Grazzini, Nucl. Phys. B 791, 1 (2008)

46. M. Grazzini, J. High Energy Phys. 0802, 043 (2008)

47. G. Ferrera, M. Grazzini, F. Tramontano, Phys. Rev. Lett. 107, $152003(2011)$

48. S. Catani, L. Cieri, D. de Florian, G. Ferrera, M. Grazzini, Phys. Rev. Lett. 108, 072001 (2012)

49. R. Hamberg, W.L. van Neerven, T. Matsuura, Nucl. Phys. B 359, 343 (1991). [Erratum-ibid. B 644, 403 (2002)]

50. R.V. Harlander, W.B. Kilgore, Phys. Rev. Lett. 88, 201801 (2002)

51. R.K. Ellis, G. Martinelli, R. Petronzio, Nucl. Phys. B 211, 106 (1983)

52. R.J. Gonsalves, J. Pawlowski, C.-F. Wai, Phys. Rev. D 40, 2245 (1989)

53. P.B. Arnold, M.H. Reno, Nucl. Phys. B 319, 37 (1989). [Erratumibid. B 330, 284 (1990)]

54. T. Gehrmann, T. Lubbert, L.L. Yang, arXiv:1209.0682 [hep-ph] 